Instituto Internacional de Investigación y Desarrollo Tecnológico Educativo INDTEC, C.A.

DOI: https://doi.org/10.29394/scientific.issn.2542-2987.2017.2.4.18.319-336

OAI-PMH: http://www.indteca.com/ojs/index.php/Revista Scientific/oai

\title{
La Praxis Docente desde la Formación Permanente
}

Autores: Yuraima Margelis Matos Universidad Nacional Experimental Simón Rodríguez, UNESR yuraimatos01@gmail.com

Trujillo, Venezuela Orlando José Cegarra Cegarra Núcleo Escolar Rural 196, Timotes cegarraorlando@hotmail.com Mérida, Venezuela

Carmen Elena Rivera Moreno Núcleo Escolar Rural 196, Timotes carmenelenarivera 17@hotmail.com Mérida, Venezuela

\section{Resumen}

Ante las exigencias y políticas que viene estableciendo el sistema educativo venezolano, se hace énfasis en la necesidad de una formación permanente del docente para que optimice su praxis educativa; más aún, cuando el docente de hoy tiene que estar en constante proceso de formación y transformación. Cabe referir que este estudio constituye parte de una investigación macro que se viene realizando en la U.E.B. El Paramito, Estado Mérida, por lo que se presenta uno de sus propósitos de investigación: develar a través de las voces de los docentes la concepción de praxis docente desde la formación permanente que reciben. Metodológicamente se abordó desde el paradigma cualitativo direccionado por la Investigación Acción Participativa, la cual surgió de un diagnóstico realizado y que llevó a describir una fase de su estudio. Para ello se realizaron mesas de trabajo, donde el diálogo prevaleció entre los actores participantes, emergiendo categorías, tales como: práctica y experiencia para un accionar, compartir de conocimientos, habilidades profesionales, las cuales al ponerlas en práctica pueden optimizar el proceso educativo en la medida que fortalecen la formación permanente del docente y con ello una mejor calidad educativa.

Palabras clave: praxis docente; formación permanente; docente. 


\title{
The Praxis Teacher from Continuing Training
}

\begin{abstract}
Related with the demands and policies that the Venezuelan system is setting, it's necessary to make emphasis in the need for continuing formation of teachers in order to optimize their educational practice; even more when teachers now day have to be in constant process of formation and transformation. It's necessary to mention that this study is part of a macro research that has been done in the U.E.B. The Paramito, Merida State, which presents one of its research purposes: to reveal through the voices of teachers conception of teaching practice from continuing training they receive. Methodologically it was addressed from the qualitative paradigm directed by the Participatory Action, which emerged from a diagnosis made and led to describe a phase research study. In this case they were made work tables, where dialogue prevailed between the actors involved, emerging categories such as were made: practice and experience for action, sharing knowledge, professional skills, which when put into practice can optimize the educational process in the as they strengthen the continuing education of teachers and thus a better quality education.
\end{abstract}

Keywords: teaching practice; continuing education; teacher. 


\section{Introducción}

En los actuales momentos, el mundo está pasando por una serie de problemas de orden social, político, cultural, económico, educativo, entre otros, razón por la cual se hace necesario que el hombre como ser bio-psico socialcultural ha de organizarse, interactuar con el medio que lo rodea e integrarse al mismo. Precisamente, esta es la columna vertebral del paradigma socio crítico reflexivo donde se conjuga el trípode: Investigación, acción y participación, la cual tiene como norte la transformación de la realidad.

La dinámica educativa que actualmente vive Venezuela lleva implícita procesos de transformación del modelo educativo en correspondencia con los propósitos de la Constitución de la República Bolivariana de Venezuela en función de producir colectivamente conocimiento para la transformación de las prácticas educativas. Para ello, se diseñó el currículo bolivariano, en tanto que orienta la relación entre las escuelas, la sociedad y el Estado, incorporando orientaciones aportadas por las y los docentes, como elemento relevante para definir la pertinencia de la educación,

Efectivamente Manterola (citado por Núñez; 2005: 148) manifiesta que "todo currículo debe tener su fundamentación epistemológica". Lo expresado con anterioridad por el autor traduce a una interpretación de tinte ontológico debido a que, en un determinado contexto la praxis educativa hace que el docente cumpla en su institución y comunidad con una serie de roles en su desempeño, los cuales lo convierten en un ente multifuncional.

Desde el enfoque epistemológico, las políticas emanadas desde el nivel central por parte del Ministerio del Poder Popular para la Educación son determinantes para que los educadores se formen a través de los congresos y colectivos, donde se conjugan los intercambios recíprocos del episteme y el cognitivismo. De acuerdo con la Ley Orgánica de Educación de la República Bolivariana de Venezuela (2009: 34), en el Art. 39 expresa que "la formación permanente es un proceso integral, continuo, que mediante políticas, planes y 
proyectos, actualiza y mejora el nivel de conocimiento y desempeño de las (los) responsables en la formación de ciudadanos y ciudadanas. Así mismo, la formación permanente deberá garantizar el fortalecimiento de una sociedad crítica, reflexiva y participativa en el desarrollo y transformación social que exige el país.

Cabe referir que este estudio se viene desarrollando desde el año 2013, debido a la apatía que se observaba por ciertos docentes quienes se sentían obligados a participar en talleres o muchas veces no asistían. En tal sentido, se realizaron mesas de trabajo de reflexión y de trabajo que llevó a los docentes involucrase más. De igual manera, es de referir que en el año 2014, el Ministerio del Poder Popular para la Educación (2015), de acuerdo a investigaciones realizadas a través de la consulta educativa emergió el sistema de investigación y formación, teniendo como uno de los alcances más importantes, la activación, de los centros locales, haciendo uso de los seminarios, congresos pedagógicos, sábados pedagógicos, foros entre otros. Cuyo tema de formación docente se derivó de las catorce líneas de investigación como necesidad de formación de los docentes. Considerando que las anteriores políticas habían fracasado, con respecto a la formación docente desde el punto de vista significativo y constructivista se refiere.

En este sentido, los investigadores desde el accionar participativo, de formación y transformación del docente del NER 196, específicamente los adscritos a la Unidad Educativa "El Paramito", en cuanto a sus roles de socializador, pedagogo, didacta, evaluativo y gerencial, se establecieron como propósito desde la reflexión y la crítica de los docentes develar a través de sus voces la concepción de praxis docente desde la formación permanente. 


\section{Sustento teórico}

\subsection{Formación docente}

Según Orozco y otros (2002: 14) "La formación docente significa construir un hombre a partir de su praxis social, de conocer su mundo y conocerse para interaccionar con su realidad y transformarla". En este sentido, los autores asumen una posición filosófica y teórica, con sus implicaciones en la práctica concreta, traducida en la formación y transformación de docentes para que se conviertan en agentes del cambio en el aspecto de la relación con el contexto, así como también en su concepción pedagógica, didáctica, gerencial y evaluativa.

En efecto, el docente al incorporarse a las actividades educativas, el mismo desempeña un rol de vital importancia en la vida escolar, por cuanto es considerado como el responsable de la docencia, donde se conjuga una multiciplicidad de funciones aunado a una gran variedad de arista de orden social, institucional, gremial e incluso político, ya que todas las actividades tanto académicas como pedagógicas dependen de las políticas emanadas del Ministerio del Poder popular para la Educación. En este sentido, la esencia de la investigación versa en la realización de conversatorios, discusiones y disertaciones donde se persigue una constante formación inherente a los docentes del NER 196, para de esta manera generar una transformación basada en sus múltiples facetas en cuanto a las actividades intra y extra escolares se refiere.

Por otra parte, no se deben obviar las teorías pedagógicas a través de dicha formación, sustentadas en un modelo de acción y formación del hombre, dirigido a una forma de producción del conocimiento, a la explicación de la realidad docente y de la problemática educativa. Orozco (2002: 2), señala que "La formación docente es un proceso permanente, que acompaña todo el desarrollo de la vida profesional", la misma se divide en formación docente inicial y continua. 
En cuanto a la Formación Docente inicial Orozco (ob.cit.: 4), tiene la finalidad de preparar profesionales capaces de enseñar, generar y transmitir los conocimientos y valores necesarios para la formación integral de las personas, el desarrollo nacional y la construcción de una sociedad más justa, la cual promoverá la construcción de una identidad docente, basada en la autonomía profesional, el vínculo con las culturas y las sociedades contemporáneas, el trabajo en equipo, el compromiso en la igualdad y la confianza en las posibilidades del proceso enseñanza y aprendizaje.

En lo que corresponde a la formación docente continua, para Orozco (ob.cit.), es entendida como desarrollo profesional y es la estrategia fundamental tanto para renovar su oficio, des esta manera debe responder a las nuevas necesidades de la sociedad, atendiendo a la complejidad de la tarea de enseñanza y de mediación cultural que realizan en sus diferentes dimensiones política, sociocultural y pedagógica. Precisamente, al conjugar los tipos de formación docente basados en la praxis educativa es bueno acotar que ésta es la vertiente de la investigación, es decir formar y transformar el rol del docente para de esta manera optimizar su desempeño, así como también mejorar el proceso de enseñanza y aprendizaje, de acuerdo a las necesidades del contexto.

Del mismo modo, es necesario enfatizar que la consulta promulgada por el Ministerio del Poder Popular para la Educación (2012), la misma tiene inherencia en la formación y transformación del docente en su praxis educativa. En efecto, de dicha formación surge la productividad diaria que el dador de clases pueda generar las actividades diarias, bien sea intra o extra escolar. De hecho, para hablar de calidad educativa que se imparte en el proceso de enseñanza y aprendizaje, se debe comenzar con la calidad en la formación docente, que contribuya a una mejor praxis docente. 


\subsection{Práctica docente}

De acuerdo con De Lella (1999: 78) "la práctica docente se considera como la acción que el profesor desarrolla en el aula, especialmente referida al proceso de enseñar y se distingue de la práctica institucional global y la práctica social docente. Por otro lado, García Cabrero y otros (2008: 76) plantean la necesidad de distinguir entre la práctica docente desarrollada en las aulas y una práctica más amplia, llevada a cabo por los profesores en el contexto institucional, denominada práctica educativa.

Al considerar la teoría sustentada por ambos autores, la misma tiene íntima relación con la calidad educativa inmersa en los procesos de enseñanza y aprendizaje donde la interacción entre el docente y discente va mucho más allá. Así mismo la praxis docente se conceptúa como la conjugación entre la investigación experimental y científica, donde está inmerso el aspecto multifuncional en su contexto de trabajo, enmarcado en el ámbito social, pedagógico, didáctico y gerencial. Desde otro ángulo, se considera la práctica educativa como una actividad dinámica, sistemática, reflexiva, la cual ocurre antes, entre y luego de cualquier planificación que haga el docente como productor, forjador y formador de generaciones.

Por otra parte, Coll y Solé (2002: 25) "señalan que la práctica educativa debe comprender el estudio de la interacción e influencia educativa, que se refiere al cómo aprenden los estudiantes con ayuda del maestro". En consecuencia, partiendo de que el individuo como ser bio psico social cultural está inmerso en un contexto rodeado de personas con características muy particulares, el docente en su rol protagónico de socializador, pedagogo, didáctico, gerente y evaluador está constantemente interactuando tanto con el personal, como con sus estudiantes y la comunidad en sí.

Así mismo, la Ley Orgánica de Educación (2009), artículo 38, expresa que la formación permanente es un proceso integral, continuo que mediante políticas, planes y proyectos, actualiza y mejora el nivel de conocimientos y 
desempeño de los y las responsables y corresponsable en la formación de sus ciudadanos y ciudadanas. La formación permanente deberá garantizar el fortalecimiento de una sociedad crítica, reflexiva y participativa en el desarrollo y transformación social que exige el país.

\section{Sustento metodológico}

Considerando el enfoque paradigmático cualitativo y crítico de la investigación es cualitativa y que se deriva de una Investigación Acción Participativa, la cual para Sandín (2003: 161), está muy orientada a la práctica educativa, siendo su fin aportar información que guie la toma de decisiones y los procesos de cambios y transformaciones. Desde una postura epistemológica el conocimiento se construye por medio de la práctica desde la propia acción de los actores sociales.

Desde esta perspectiva los investigadores y participantes, realizaron una reunión que le permitió organizarlos en mesa de trabajo propiciando la discusión de grupos dirigidos, que de acuerdo con Ulin (2006: 82) "consiste en usar la interacción del grupo para generar datos e interpretaciones que serían menos accesibles sin la interacción encontrada en un grupo". La fase clave en esta estrategia es la interacción del grupo. Los grupos de discusión dirigidos dependen tanto del intercambio de ideas entre participantes como de las respuestas a preguntas específicas del entrevistador. En este caso los investigadores fungieron de moderador o facilitador del proceso grupal, para orientar y dirigir las preguntas que les permitieran a los docentes expresarse libremente.

Información que se sistematizó, organizó, codificó y categorizó. En este sentido se construyeron categorías, que para Anguera (1988: 29), es un intento de agrupar la información recogida sobre la base a unos casos preestablecidos y en otros que emergen en el momento de la recogida de la información, las cuales que se presentan a continuación. 


\section{Categorías emergentes.}

Develar la concepción de la praxis docente desde los actores sociales, en este caso de los docentes de la U.E. El Paramito, implica todo un proceso epistemológico que permite conocer desde el decir y hacer de estos actores formadores en su praxis educativa. Freire (1978: 121) señalaba que somos seres programados, pero no determinados. Entonces, pensar la dinámica del proceso de conocer que resulta de la praxis de los docentes sobre la realidad exige plantear, a su vez, el proceso de inacabamiento e incompletud de los hombres y mujeres insertos en esas realidades también inacabadas y, por lo tanto, cambiantes. En este sentido, se puede decir que, los hombres y mujeres son totalidades dinámicas expresadas en la articulación de lo afectivo, cognitivo, volitivo e imaginativo, que le llevan a ver su realidad según el contexto en que se desenvuelven y el momento histórico que viven.

4.1. Práctica y experiencias para un accionar, tal como lo señala el docente especialista 7, "a mí, praxis me suena como un grupo de experiencias que se relacionan con la práctica". Posteriormente, la docente 5 pidió la palabra y señaló "praxis tiene que ver con lo que el docente hace y, en su accionar está su formación". Para Romero, Tobo, Jinete, y Lindo (2006: 78), conciben a la práctica como la actividad visible-material de las personas; es decir, el conjunto de actuaciones de los actores sociales con que pretenden satisfacer, de manera directa o indirecta, sus necesidades y que implican unas acciones operativas, es decir, tareas secuenciadas orientadas por un sentido, que genera efectos en los actores e impacto en el medio social y natural. A la vez de actitudes hacia lo que dicen y piensan y, comportamientos, dirigido a las reacciones emotivas y formas de movimiento físico del cuerpo.

Así mismo, Bolívar (2007: 46), señala que la praxis constituye una categoría de interpretación de la práctica social que refiere a la reflexión sobre la práctica y al mismo tiempo la intervención en la práctica, traducida en 
construcción de la realidad. En virtud de ellas se transforman los objetos 0 fenómenos, artificiales o naturales, o se generan efectos en los actores sociales durante sus interacciones, a través de actividades que conforman situaciones sociales y se impacta en éstas. En este sentido la praxis educativa, es vista como la práctica de la relación del sujeto cognoscente con el sujeto cognoscible.

4.2. La praxis docente vista como compartir conocimientos, tal como lo expresa la docente 3, "a mi modo de ver entiendo, que praxis viene de la palabra práctica, pero en la práctica debe haber algún conocimiento y de cualquier modo, ambos tienen que ver con la formación en el caso de nosotros que somos docentes". Por su parte, la docente 10 dijo: "la praxis docente es accionar para compartir conocimientos entre docente y estudiantes", por otra parte, el Docente 9, refirió: "la praxis docente implica un proceso abierto y flexible que lleva a la construcción del conocimiento entre el docente y estudiantes".

Expresiones que reflejan el compartir conocimientos en la construcción dialógica de espacios y tejidos de participación en la construcción de saberes. Es lo que en palabras de Bolívar (2007: 46), es el reconocimiento del accionar con el otro, de compartir abiertamente conocimientos y saberes en la diversidad que representa la praxis de realización humana. De igual manera, Paquay, Altet, Charlier y Perrenoud (Coords; 2005: 39), señalan que durante la práctica del docente se articulan de manera funcional en el curso de la acción, conocimientos y habilidades profesionales que lo llevan a construir y reconstruir conocimientos con los estudiantes.

4.3. La praxis docente como habilidades profesionales; la cual en palabras de la Docente 2, refiere "para mí son las habilidades que posee todo docente en su accionar educativo, pues debemos no sólo poseer conocimientos, sino 
también ponerlos en práctica, a la vez que mantenemos una actitud afectiva con los niños y niñas", a lo que el Docente 7 , señala "estamos reflexionando sobre la praxis educativa, donde podemos visualizar las habilidades que como docente tenemos que poner en práctica dentro y fuera del aula de clase, para hacer del proceso educativo más efectivo".

Desde las voces de los actores participantes, se aprecia el realce de la puesta en práctica de las habilidades profesionales por parte del docente, lo cual para Paquay y otros (2005: 41), las habilidades profesionales son "el conjunto de conocimientos, procedimientos y el saber-estar, pero también el hacer y el ser necesarios para el ejercicio de la profesión docente". Desde esta concepción, las habilidades son de orden cognitivo, afectivo, y práctico, por cuanto posee conocimientos y formación para los cuales debe considerar la parte afectiva, el ser de cada estudiante para ponerlos en práctica desde el hacer en conjunto estudiante y docente.

Desde las categorías conformadas en función de las expresiones de los docentes participantes, es notorio señalar que la discusión, inherente a la praxis, llevó al análisis de concepciones versadas en las ciencias sociales, partiendo de Carlos Marx, hasta Kurt Lewin, sin dejar entrever la pedagogía práctica de Paulo Freire. Haciendo énfasis en que las diversas posturas enunciadas son necesarias reflexionarlas para hacer de la praxis educativa un momento de placer. De allí que los lleve a formarse día a día para dar respuesta a las exigencias y políticas que establece el sistema educativo y el país.

En síntesis y producto del análisis y reflexión de los actores educativos como de los investigadores, se puede decir que la praxis docente es la puesta en práctica de experiencias, conocimientos y habilidades para un accionar activo entre docente y estudiantes, a partir de la construcción dialógica de saberes y conocimientos, en donde todos aprenden en la medida que se genera la interacción, afectiva, cognitiva y práctica que debe desempeñar 
efectivamente el docente hacia la transformación educativa que requiere el país.

Reflexión: Ya terminando la jornada, se pudo evidenciar que, teniendo como testigo fiel, la participación muy objetiva de cada uno de los actores sociales, donde se evidenció la claridad y entendimiento de la actividad. Visualizándose el respeto por las ideas de cada participante, así como la sinceridad de cada uno, al extremo de poder apreciar la expresión del docente de aula 14, quien señaló "actividades así, son las que se necesitan, porque el aprendizaje le llegó a cada docente, sin menospreciar los congresos casi no se aprende nada, porque se convierten en monólogos y casi no dan cabida para la participación.

Tanto es así que Colomina y otros (2001: 34) indican que en "la interactividad se deben considerar situaciones surgidas antes, durante y después de las actividades escolares, así como aprendizajes generados por los estudiantes y su inferencia en la actividad social, cognitiva y las acciones del profesor para que ocurran". En suma, cada realidad va a incidir en el contexto donde se desenvuelva el docente, de allí surge el rol transformador de la misma, la cual se traduce en sus múltiples funciones.

Para finalizar se puede decir, que las mesas de trabajo realizadas permitieron la reflexión del porqué de la formación permanente y los beneficios que direcciona una mejor praxis educativa, crítica y reflexiva. Discusión que llevó no sólo a develar lo qué significa praxis docente, sino que generó la construcción de categorías desde las voces de los mismos actores participantes, llegando a generar el concepto de praxis educativa, tal como se puede apreciar en la interpretación de los investigadores y, que se refleja en el siguiente gráfico. 
Gráfico 1. Concepción de la praxis docente.

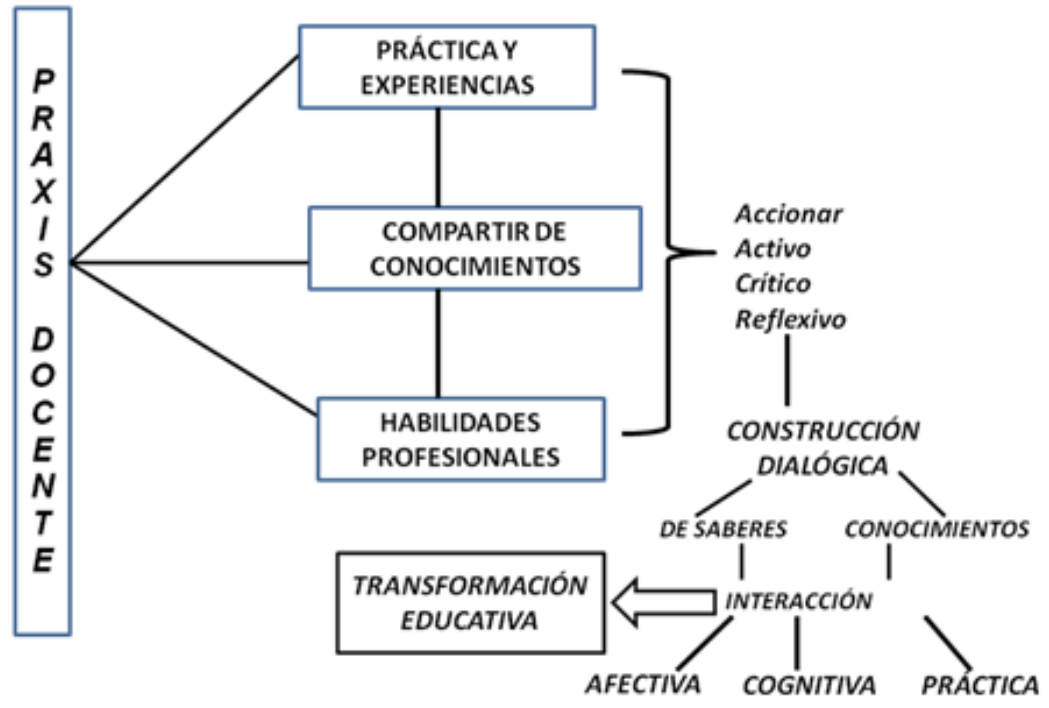
los objetivos, a través del diálogo, la dialéctica, la cual, conlleva al fortalecimiento y logro de lo que queremos controlar. En este sentido, para 
Paulo Freire, la transformación del educador es inherente al uso de un buen entendimiento en el cual, se pueden generar discusiones, conversatorios entre otros, de acuerdo a las líneas de orientación que emana el sistema educativo. Considerando que una de las políticas educativas hoy día es que el docente se forme para que forme desde una postura onto-epistémica que lleve a la transformación social, aunada a una educación emancipadora.

En este sentido, se espera que los docentes promuevan el reaprendizaje desde su praxis, que los lleve a transformar la práctica pedagógica, favoreciendo así la educación integral de los educandos, y su proceso de liberación y autonomía. Por lo tanto, es necesario que el docente se valga de diversas estrategias que lleven al diálogo, abiertos a relacionarnos con el otro, a trabajar en equipo, a respetar la diversidad de pensamiento. Un diálogo que propicie la crítica para discernir y mantener posturas fijas ante situaciones que se le presenten. Considerando no sólo lo cognitivo sino también el hacer y actuar como los sentimientos, actitudes, emociones, para obtener la educación que tanto queremos.

\section{Referencias}

Coll, C., y Sollé, I., (2002). La Interacción Profesor Alumno en el Proceso de Enseñanza Aprendizaje. Psicología de la Educación. Madrid. España.

Colomina y otros (2001). El análisis de la actividad discursiva en la interacción educativa. Algunas opciones teóricas y método lógicas. Madrid. Editorial Alianza.

Constitución de la República Bolivariana de Venezuela (1999). Gaceta Oficial № 36.860. Venezuela.

De Lella, C. (1999). Modelos y tendencias de la formación docente. Consultado el 24 de Febrero de 2013. Recuperado de: http://www.oei.es/historico/cayetano.htm 
García Cabrero y otros, (2008). Las aproximaciones teórico-metodológicas en torno a la construcción de un modelo de evaluación para la práctica educativa. México.

Freire, P. (1997). Pedagogía Crítica. 8va edición. Sao Paolo.

Freire, Paulo (2006). Pedagogía de la autonomía. Saberes necesarios para la práctica educativa. Traducido por Guillermo Palacios. Undécima edición en español. México: Siglo XXI.

Ley Orgánica de Educación (2009). Gaceta Oficial № 5929 Extraordinario, de fecha de 15 de agosto de 2009. Caracas-Venezuela.

Ministerio del Poder Popular para la Educación (2012). Diseño Curricular. Caracas, Venezuela.

Ministerio del Poder Popular para la Educación (2015). Orientaciones Pedagógicas año escolar 2014- 2016. Caracas, Venezuela.

Orozco y otros, (2002). Formación y Práctica Docente en el Medio Rural. México. Editorial Plaza y Valdés.

Paquay, L. (2005). La formación profesional del maestro estrategias y competencias. Editorial FCE. México.

Romero, H., Tobis, M., Jinete, M. y Lindo, M. (2006). La praxis profesional del docente en formación. En Revista Iberoamericana de Educación. OEI. No $10 / 5$.

Sandín, M. (2003). Investigación cualitativa en Educación. Fundamentos y tradiciones. Madrid: McGraw-Hill.

Ulin, P. (2006). Investigación aplicada en salud pública. Métodos cualitativos. España: Editorial Washington D.C. 


\section{Yuraima Margelis Matos \\ e-mail: yuraimatos01@gmail.com}

Nacida en Venezuela. Licenciada en Educación

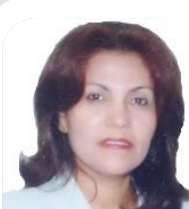
Integral, Mención Matemáticas, Magister en Ciencias de la Educación. Mención Investigación Docencia. Magister en Tecnología y Diseño educativo, Magister en Educación Robinsoniana, Dra. En Ciencias de la Educación. Docente investigador en la Universidad Nacional Experimental Simón Rodríguez, (UNESR) Núcleo Valera, Edo. Trujillo (Jubilada). Participante de la Línea de investigación: Investigadores en acción Social (IAS), Facilitadora en cursos de metodología, Seminario de investigación, Valores, Servicio Comunitario, Proyecto de Investigación, administración de la educación Básica, Ética y valores en la investigación, gerencia educativa, entre otros cursos, en las Universidades: Simón Rodríguez, Valle del Momboy, Rafael María Baralt (Especialidad, Maestría y Doctorado). Jurado evaluador y tutora de Trabajo Especial de Grado y Tesis, Evaluadora de trabajos de ascenso, artículos arbitrados en Revistas nacional e internacional. Actualmente miembro del PEII, NIVEL B. 


\section{Orlando José Cegarra Cegarra \\ e-mail: cegarraorlando@hotmail.com}

Nacido en Venezuela. Licenciado en Educación, Mención Inglés. (ULA). Especialista en Evaluación Educacional. (VALLE DE MOMBOY). T.S.U. en Educación Integral. (IUTEMBI). Msc en Gerencia de la Administración Básica. (UNERMB). Doctorado en Educación (ESCOLARIDAD) (UNERMB). Docente de Inglés en Media General. Docente de Aula en el Núcleo Escolar Rural 196. Sub Director del NER196. Director del Ner 196. Supervisor del ner196.Docente en la Misión Sucre. Facilitador de talleres, ponente, tutor, evaluador de trabajo de Grado. 


\section{Carmen Elena Rivera Moreno}

e-mail: carmenelenarivera 17@hotmail.com

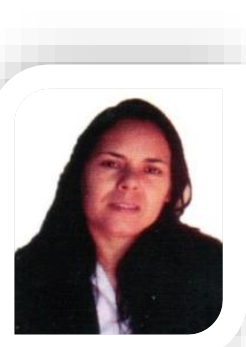

Nacida en Venezuela. Licenciada en Educación Integral (UNIOJEDA) Magister Scientiarum en Administración de la Educación Básica. (UNERMB). T.S.U. Educación Integral (IUTEMBI). Doctorado en Educación (Escolaridad Aprobada) (UNERMB). Docente de Aula NER 196 Municipio Miranda estado Mérida. Supervisora Municipio Miranda Estado Mérida. Colaboradora del CLIFM Municipio Miranda. Docente en la Misión Sucre. 